\title{
A Note on Extensions: Admissible Rules via Semantics
}

\author{
Jeroen Goudsmit*
}

October 19, 2012

\begin{abstract}
Any intermediate logic with the disjunction property admits the Visser rules if and only if it has the extension property. This equivalence restricts nicely to the extension property up to $n$. In this paper we demonstrate that the same goes even when omitting the rule ex falso quod libet, that is, working over minimal rather than intuitionistic logic. We lay the groundwork for providing a basis of admissibility for minimal logic, and tie the admissibility of the Mints-Skura rule to the extension property in a stratified manner.
\end{abstract}

Keywords: admissible rules, minimal logic, disjunction property, extensions of Kripke models

The admissible rules of a theory are those rules under which the theory is closed. Derivable rules are admissible. For classical propositional logic, this is the whole story. For intuitionistic propositional logic (IPC) - and minimal logic — it is not.

Friedman (1975, Problem 40) conjectured admissibility for IPC to be decidable, as has been confirmed by Rybakov (1984). De Jongh and Visser conjectured that the Visser rules form a basis of admissibility for IPC, that is to say, all admissible rules of IPC become derivable after adjoining the Visser rules. Rozière (1992) and Iemhoff (2001b) independently confirmed this. Again independently, Skura (1989) demonstrated that IPC is the sole intermediate logic that admits a restricted form of the Visser rules.

At the Pisa Proof Theory workshop of 2012 George Metcalfe gave a tutorial on admissible rules. As has become standard practice, Metcalfe mentioned Lorenzen (1955) as the first place where admissible rules where studied an sich. Jan von Plato objected that Johansson (1937) already discussed them. Odintsov and Rybakov (2012) proved admissibility for minimal logic to be decidable. In this paper we lay the groundwork for studying all admissible rules of Johansson's minimal logic, with the eventual goal of providing an explicit basis of admissibility.

This paper aims to provide uniformity to some of the literature regarding admissible rules for logics above minimal logic. We make several observations, many of which not elsewhere available in the generality stated here. Although this paper contains novel results, most notably the semantic characterization of admissibility for an adaptation of the rules studied by Skura, its main purpose is to provide a unified approach to the study of admissible rules over minimal logic.

\footnotetext{
* Support by the Netherlands Organisation for Scientific Research under grant 639.032.918 is gratefully acknowledged. This paper will appear in "Symposium on Logical Foundations in Computer Science 2013", edited by Sergei Atermov and Anil Nerode, to be published in the Springer-Verlag Lecture Notes in Computer Science.
} 


\section{Preliminaries}

We first fix some basic notation. Many definitions are fairly straightforward adaptations of their well-known intuitionistic counterparts.

\section{Definition (Propositional Language)}

The language of propositional formulae is defined as follows, starting from a fixed countably infinite set of propositional variables Var. A formula is said to be atomic if it consists solely of a variable or falsum, the set of atomic formulae is denoted as Atom.

$$
\mathcal{L}::=\operatorname{Var}|\perp| \mathcal{L} \wedge \mathcal{L}|\mathcal{L} \vee \mathcal{L}| \mathcal{L} \rightarrow \mathcal{L}
$$

We will denote formulae by captital Latin letters at the beginning of the alphabet, and use greek capitals to refer exclusively to finite sets of formulae. For greater convenience we write $\Gamma \Rightarrow \Delta$ to mean $\Lambda \Gamma \rightarrow \bigvee \Delta$, that is to say, the conjunction of all formulae in $\Gamma$ implies the disjunction of all formulae in $\Delta$. We will only use this notation when both $\Gamma$ and $\Delta$ are non-empty. This definition of a Kripke model differs in one important regard from the standard definition as given for instance by Troelstra and Dalen (1988). The difference is in that a valuation can determine whether $\perp$ is to hold, analogous to the definition of Došen (1989), whereas in a Kripke model of IPC this is fixed. This to ensure completeness for minimal logic, in which $\perp$ does not derive everything.

\section{Definition (Kripke Model)}

A Kripke model is a pair $K=\langle K, v\rangle$ where $K$ is a partial order and $v$ (the valuation) is a monotone map $v: K \rightarrow \mathcal{P}$ (Atom). We define a relation $\Vdash$ (forces) between $K$ and $\mathcal{L}$ inductively as follows

$$
\begin{array}{ll}
k \Vdash A & :=k \in v(A) \text { for atomic } A \\
k \Vdash A \wedge B & :=k \Vdash A \text { and } k \Vdash B \\
k \Vdash A \vee B & :=\quad k \Vdash A \text { or } k \Vdash B \\
k \Vdash A \rightarrow B & :=\quad l \Vdash B \text { whenever } l \Vdash A \text { for all } k \leq l
\end{array}
$$

The model $K$ is said to be rooted if $K$ has a least element, and $K$ is strict when $k \Vdash \perp$ holds for no $k \in K$. We say that $K$ is finite when $K$ is finite and $v$ maps but finitely many atoms to a non-empty upset. As usual, we write $K \Vdash A$ to mean that $k \Vdash A$ for all $k \in K$. For convenience we also write $K \Vdash x$ when $x \subseteq \mathcal{L}$ to mean that $K \Vdash A$ for all $A \in x$. The theory of $K$, written Th $K$, equals the set of formulae $A$ such that $K \Vdash A$.

Kripke models can be endowed with a topology, the Alexandroff topology, where opens are exactly upsets. Using the thus inherited topology we can define a sensible notion of maps as follows. These kinds of maps have been called p-morphisms and bounded morphisms, we will simply call them maps (of Kripke models). From the definition it naturally follows that for any map $f: K \rightarrow L$ we have $\operatorname{Th} L \subseteq \operatorname{Th} K$.

\section{Definition (Maps of Kripke Models)}

A map $f:\langle K, v\rangle \rightarrow\langle L, w\rangle$ between Kripke models is an open and continuous function such that $w \circ f=v$.

We often place models next to one another, below a formal definition. 


\section{Definition}

Given a set of Kripke models $\mathcal{K}=\left\{K=\left\langle K, v_{K}\right\rangle \mid K \in \mathcal{K}\right\}$ we define their disjoint union as

$$
\coprod \mathcal{K}:=\left\langle\coprod \mathcal{K}, \bigcup_{K \in \mathcal{K}} v_{K}\right\rangle
$$

In order to be as generic as possible, and to not get involved with the intricacies of axiomatizations of the logics at hand, we use the notion of a consequence relation. Rybakov (1997) already used consequence relations in the context of admissible rules, we shall do the same. We will use the formulation of Cintula and Metcalfe (2010), where a consequence relation is concerned with multiconclusion rules. ${ }^{1}$ Multi-conclusion rules allow for a more succinct notation of the rule schemes we will use later on, and help us steer clear of some obstacles concerning the disjunction property, see Citkin (2012).

First, a rule is an ordered pair of finite sets of formulae, written $\Gamma / \Delta$. A consequence relation is simply a set of rules satisfying certain sensible properties. When reading the following definition, think of the relation $\vdash$ defined as $\Gamma \vdash \Delta$ iff there is a (minimal logic) proof of some $A \in \Delta$ with assumptions in $\Gamma$.

\section{Definition (Consequence Relation)}

A consequence relation (or logic) consists of a relation called derivability, denoted $\vdash$, between finite sets of formulae subject to the following axioms, where $A$ is a formula and $\Gamma, \Theta, \Delta, \Pi$ are finite sets of formulae.

reflexivity $\quad A \vdash A$;

monotonicity $\quad$ if $\Gamma \vdash \Delta$, then $\Gamma, \Theta \vdash \Delta, \Pi$;

transitivity $\quad$ if $\Gamma \vdash \Delta, A$ and $A, \Theta \vdash \Pi$, then $\Gamma, \Theta \vdash \Delta, \Pi$;

structurality $\quad$ if $\Gamma \vdash \Delta$, then $\sigma(\Gamma) \vdash \sigma(\Delta)$.

We extend the notation to infinite sets on the left by defining $x \vdash \Delta$ to mean that there exists a finite $\Gamma \subseteq x$ such that $\Gamma \vdash \Delta$. When one of the sets is a singleton we omit braces, and if it is empty we omit it entirely. A formulae $A$ is said to be a theorem of this consequence relation if $\vdash A$. A logic is consistent when not all formulae are theorems.

Given any set of formulae $x$ and any consequence relation $\vdash$ one can form a new consequence relation $\vdash_{x}$ where $\Gamma \vdash_{x} \Delta$ holds if and only if $x+\Gamma \vdash \Delta$. We say that $x$ is a theory if it contains all theorems of $\vdash_{x}$. Moreover, given a consequence relation $\vdash$ one can form the consequence relation of admissibility. Roughly said, a rule is admissible when for each substitution we know that if its assumptions are theorems under the substitution, then one of the conclusions must be a theorem under the same substitution.

\section{Definition (Admissible Rule)}

Let $\vdash$ be a consequence relation and $\Gamma / \Delta$ a rule. We say that $\Gamma / \Delta$ is admissible, denoted $\Gamma \sim \Delta$ when for each substitution $\sigma$, if $\vdash \sigma(A)$ for all $A \in \Gamma$, then $\vdash \sigma(A)$ for some $A \in \Delta$.

\footnotetext{
${ }^{1}$ Note that they call the definition below a "finitary structural multi-conclusion consequence relation". We call this simply a consequence relation or logic.
} 


$\begin{array}{ccc}\frac{\Gamma \vdash A}{\Gamma \vdash A \wedge B} & \frac{\Gamma \vdash A \wedge B}{\Gamma \vdash A} & \frac{\Gamma \vdash A \wedge B}{\Gamma \vdash B} \\ \frac{\Gamma \vdash A}{\Gamma \vdash A \vee B} \quad \frac{\Gamma \vdash B}{\Gamma \vdash A \vee B} & \frac{\Gamma \vdash A \vee B \quad}{\Gamma, \Gamma \vdash C} \quad B, \Gamma \vdash C \\ \frac{\Gamma, A \vdash B}{\Gamma \vdash A \rightarrow B} & \frac{\Gamma \vdash A \rightarrow B}{\Gamma \vdash B}\end{array}$

Figure 1: Closure properties of $\vdash$

The thus defined relation $\sim$ of admissibility is a consequence relation. By structurality, $\sim$ contains $\vdash$, so reflexivity is clear. All other properties are a simple matter of verification. A rule is said to be admissible for $x$ whenever it is admissible for $\vdash_{x}$.

It is important to keep in mind that a logic need not satisfy the deduction theorem, that is, $\Gamma+A \vdash B$ need not be equivalent to $\Gamma \vdash A \rightarrow B$, even when the logic is an extension of minimal logic. Likewise, when a rule is admissible for a given logic, it need not be admissible for an extension. In the following, we will let $\vdash$ stand for any logic which contains minimal logic, satisfying the close properties of Fig. 1, satisfying the deduction theorem.

\section{Extensions of models}

An extension of a Kripke model is that same model, adjoined with a least element and a choice of valuation there. In this section we investigate when a given model of a theory has an extension satisfying the same theory. This is interesting in and of itself, but it also has applications for admissible rules. The characterization that is to be given at the end of this section suggests a particularly nice schema of admissible rules, namely the de Jongh rules. With some additional bookkeeping one can use this characterization to prove, for instance, that the de Jongh rules form a basis of the Gabbay-de Jongh logics of Gabbay and de Jongh (1974), as has been done in Goudsmit and Iemhoff (2012). Here we show how these results are actually more general, in that they help towards providing a basis of admissibility of minimal logic. As we do not attain this goal here, we omit the bookkeeping to make the material more digestible.

Let us now first introduce notation for extensions. What the following definition comes down to is that the model $K / x$ is $K$ with a node (named $\underline{x}$ ) placed below it, which forces precisely the atomics $x$ contains. For greater convenience we will often write $K / x$ even when $x$ contains nonatomic formulae, this is understood to denote $K /(x \cap$ Atom $)$.

\section{Definition (Extension)}

Let $K=\langle K, v\rangle$ be a Kripke model and $x \subset$ Th $K$ be a set of atoms. We define the extension of $K$ over $x$, denoted $K / x$, as follows.

$$
K / x=\left\langle K_{x},\left(k \in K_{x} \mapsto v(A) \text { if } k \in K \text { and } x \text { otherwise }\right)\right\rangle
$$

Here $K_{x}$ is the partial order with underlying set $K+\{\underline{x}\}$ ordered by $k \leq l$ if and only if $k \leq l$ holds in $K$ or $k=\underline{x}$. 
The following characterization is fairly straightforward and can be proven with almost no effort at all. It does look fairly familiar to the inductive characterization of the Aczel Slash as given in Smoryński (1973, Theorem 5.1.18), and this is no coincidence. When we take $K$ to be the canonical model of some theory $x$ as in Definition 9, then this characterization is identical, as is can be readily seen in the presence of Lemma 2. Another observation: if $K \Vdash A \rightarrow B$ but $K \forall A$ then $K / x \Vdash A \rightarrow B$, which clarifies the importance of Definition 11 below. Later on we will formulate some constraints on theories $x$ given models $K$ under which Th $K / x$ actually equals $x$. We will give an exact characterization when given a model $K$ and a theory $x$ there exists some extension $K / y$ such that Th $K / y \supseteq x$.

\section{Lemma (Forcing of Extensions)}

Let $K$ be any Kripke model and let $x \subseteq$ Th $K$ be arbitrary. The following hold:

$$
\begin{array}{lll}
K / x \Vdash C & \text { iff } & x \ni C \text { for atomic } C \\
K / x \Vdash A \wedge B & \text { iff } & K / x \Vdash A \text { and } K / x \Vdash B \\
K / x \Vdash A \vee B & \text { iff } & K / x \Vdash A \text { or } K / x \Vdash B \\
K / x \Vdash A \rightarrow B & \text { iff } & K \Vdash A \rightarrow B \text { and if } K / x \Vdash A \text { then } K / x \Vdash B
\end{array}
$$

A logic is said to have the disjunction property when each derivable disjunction has a derivable disjunct. See Chagrov and Zakharyashchev (1991) for a wonderful and comprehensive survey of intermediate logics and the disjunction property. The above characterization shows that the theory of each extension satisfies this disjunction property. So when we seek theories $x$ such that Th $K / x=$ $x$ holds, $x$ had better satisfy the disjunction property too.

To smoothen proofs we use a generalized form of the disjunction property, the idea of being saturated in something else. Note that a set of formulae has the disjunction property if it is saturated in itself, in which case we call it saturated. This is one of the many places where one could introduce further bookkeeping by restricting the formulae considered to some set, for instance the set of atomic formulae. For details regarding this one can consult Goudsmit and Iemhoff (2012), this is the last we speak of it.

\section{Definition (Saturated Set)}

Let $x \subseteq y$ be sets or formulae. We say that $x$ is saturated in $y$, written $x \preccurlyeq y$, whenever

$$
x \vdash \bigvee \Delta \text { entails } y \cap \Delta \neq \emptyset \text { for all non-empty finite } \Delta \text {. }
$$

Each theory can be extended to a saturated set, avoiding a chosen formula outside of this set. This is akin to a basic fact of lattices: given a filter and an ideal with empty intersection, there exists an extension of the filter to a prime filter, which does not intersect the ideal (see e.g. Johnstone, 1982, section 2.3). Let us mention three small results. The first is an immediate corollary of Lemma 3 and 4 of Goudsmit and Iemhoff (2012), the other two follow from the first. The final corollary comes in handy in proving that our canonical model works well, and it is also crucial in Lemma 4.

\section{Corollary}

Let $x$ be saturated in $z$. There exists a saturated set $y$ such that $x \subseteq y \subseteq z$.

\section{Corollary (Negative Saturation Lemma)}

Let $x$ and $\Delta$ be sets of formulae such that $x \not \forall \bigvee \Delta$. Now there exists a saturated set extending $x$ not intersecting $\Delta$. 


\section{Corollary}

Let $x$ be a set of formulae and let $A$ and $B$ be formulae such that $x \forall A \rightarrow B$. There exists a saturated set extending $x$ such that $z \ni A$ and $z \not \supset B$.

Let us now define the canonical model. Not to prove completeness, although it is a natural byproduct, but to link the disjunction property to a semantic property. Our canonical model is analogous to that of Došen (1989, Definition 9). It is also similar to the model of Aczel (1968), but it differs in several regards, most importantly in that his model is strict.

A theory is said to be consistent when its associated logic is. This notion of consistency is sufficient to ensure the existence of (consistent) saturated sets above a consistent theory due to the Negative Saturation Corollary 2, which we need to make the model a bona fide model. When the logic at hand would be some intermediate logic, then any theory must contain $\perp \rightarrow A$ for any $A$. This ensures that a consistent theory does not contain $\perp$, so the canonical model under intermediate logics would always be strict, as desired.

\section{Definition (Canonical Model)}

Let $x$ be a consistent theory. The canonical model of $x$, denoted by can $x$, is defined as the Kripke model

$$
\operatorname{can} x:=\langle\{y \supseteq x \mid y \text { saturated }\}, y \mapsto y \cap \text { Atom }\rangle \text {. }
$$

\section{Lemma}

For any theory $x$ we have Th $\operatorname{can} x=x$.

Proof If $x \vdash C$ then every saturated extension contains $C$. Conversely, if $x \not C$ there is a saturated extension which does not contain $C$. By structural induction along $C$ we prove that for any $y \in \operatorname{can} x$ we have $y \Vdash C$ if and only if $y \ni C$. For atomic formulae this holds by definition. The conjunction and disjunction cases follow readily from induction and saturation. We are left with the implication case, where $C=A \rightarrow B$.

From right to left, suppose that $y \ni A \rightarrow B$ and let $z \supseteq y$ be a saturated set. If $z \Vdash A$ then $z \ni A$ by induction, whence $y \subseteq z \ni B$ by saturation, induction now finished the job. To prove the converse, suppose that $y \not \supset A \rightarrow B$. This yields a saturated $z \supseteq y$ such that $z \ni A$ but $z \not \supset B$ by Corollary 3 . The desired follows by induction.

\section{Corollary (Completeness)}

For any theory $x$, if $K \Vdash A$ for all models $K$ with $x \subseteq \operatorname{Th} K$ then $x \vdash A$.

We can characterize the disjunction property via the following semantic property. This has already been proven by Maksimova (1986, Theorem 1 and 2). The proof below is quite similar, in that it uses the same core idea, and it nicely illustrates how little changes when moving to minimal logic.

\section{Theorem}

A theory $x$ has the disjunction property if and only if for every model $K \Vdash x$ there is a rooted model $L \Vdash x$ and a map $K \rightarrow L$.

Proof The implication from right to left is fairly straightforward. Suppose $x$ does not have the disjunction property. This gives some $\Delta$ such that $x \vdash \bigvee \Delta$ but for no $A \in \Delta$ we have $x \vdash A$. Via 
completeness this ensures us models $K_{A} \Vdash x$ such that $K_{A} \Vdash A$. Consider then $K:=\coprod_{A \in \Delta} K_{A}$, by assumption we have a rooted model $L \Vdash x$ and a map $K \rightarrow L$. This entails that Th $L \subseteq \operatorname{Th} K$. Observe that $L \Vdash x \vdash \bigvee \Delta$, whence $L \Vdash A$ for some $A \in \Delta$ because $L$ is rooted. This in turn entails that $K \Vdash A$ and so $K_{A} \Vdash A$, a clear contradiction.

Let us now focus on the other implication. Let $K$ be a Kripke model of $x$. Now consider the model $L:=(K+\operatorname{can} x) / x$, and see that the inclusion from $K$ to $L$ is a map. We will prove that $x \ni C$ if and only if $L \Vdash C$. We proceed via Lemma 1, and there is only some work to be done in the implicative case. Note that $L \Vdash A \rightarrow B$ iff $K+\operatorname{can} x \Vdash A \rightarrow B$ and if $L \Vdash A$ then $L \Vdash B$. It is clear that Th $\operatorname{can} x+K=$ Th $\operatorname{can} x=x$. By induction we know that $L \Vdash A$ and $L \Vdash B$ to be equivalent to $x \vdash A$ and $x \vdash B$. The burden of proof has completely dissolved, keeping in mind modes ponens.

Consider again a model $K$ of a particular theory $x$. When we can find a theory $y \supseteq x$ such that Th $K / y=y$ holds we know that an extension of $K$ forcing $x$ exists. Such a theory $y$ is, in a way, a saturated approximation of the model $K$ containing $x$. We are interested in the "best" such approximation, that is to say, a saturated extension containing $x$ such that every larger saturated extension overshoots $K$. This idea is captured by the notion of a tight predecessor. The definition first took form in Iemhoff (2001b, Section 2.1.1) where it pertained to models, and was later adopted by Jeřábek (2005, Definition 3.2) to suit modal logic. Goudsmit and Iemhoff (2012, Definition 11) adapted the idea to theories, and it is this definition we use here.

\section{Definition (Tight Predecessor)}

Let $x$ and $z$ and be sets of formulae. We say that $x$ is a tight predecessor of $z$ when $x$ is saturated, $x \subseteq z$ and for each saturated set $y \supset x$ we have $z \subseteq y$.

\section{Definition (Vacuous Implications and Assumptions)}

Let $x$ be a set of formulae. Define the following:

$$
\begin{aligned}
\mathrm{I}(x) & :=\{A \rightarrow B \mid \text { for formulae } A \text { and } B \text { such that } x \ni A \rightarrow B \text { but } x \not \ngtr A\} \\
x^{\mathrm{a}} & :=\{A \mid \text { for some } B \text { we have } x \ni A \rightarrow B\}
\end{aligned}
$$

\section{Lemma}

Let $K$ be a Kripke structure, let $x \subseteq$ Th $K$ be arbitrary and let $y$ be a set of implications. If $y^{\mathrm{a}}$ does not intersect Th $K$ and $y \subseteq \operatorname{Th} K$ then $K / x \Vdash y$. In particular, I (Th $K) \subseteq \operatorname{Th} K / x$.

We can now show that a tight predecessor of a model contains all information of the theory of the extension. In ibid., Lemma 9 the intermediate case was treated, here we consider any extension of minimal logic. The proof below uses the characterization of extensions, which makes it a little smoother than the original. Note that to prove the equivalence for a formulae $C$, one needs only knowledge of structurally smaller formulae. The additional constraint about containing $\mathrm{I}(\mathrm{Th} K)$ may be dropped, as it can be shown to always hold.

\section{Lemma (Extension Lemma)}

Let $K$ be any model, and let $x$ be a tight predecessor of Th $K$ containing I (Th $K$ ). Now Th $K / x=x$ holds. 
Proof We prove that $K / x \Vdash C$ iff $x \ni C$ by structural induction along $C$. Only the implication case is interesting, the other cases are either immediate or follow from induction and the rules under which we assumed $\vdash$ to be closed. We know that $K / x \Vdash A \rightarrow$ if and only if $K / x \Vdash B$ whenever both $K \Vdash A$ and $K / x \Vdash A$.

Let us first go from right to left. If $x \ni A \rightarrow B$ and $K / x \Vdash A$ then $x \ni A$ by induction, so $x \ni B$ by assumption. Induction yields $K / x \Vdash B$ as desired.

Now for the other direction, suppose that $K \Vdash A \rightarrow B$ and that if $K / x \Vdash A$ then $K / x \Vdash B$. Furthermore assume that $x \not \ngtr A \rightarrow B$. We will derive a contradiction from these assumptions. By Corollary 3 , there is a saturated extension $y \supseteq x$ such that $y \ni A$ and $y \not \supset B$. There are two cases, either $y=x$ or $y \supset x$.

In the former case we know $x \ni A$ and $x \not \ngtr B$, so induction yields $K / x \Vdash A$ and $K / x \Downarrow B$.

In the latter case first observe that Th $K \subseteq y$. If $K \Vdash A$ then $K \Vdash B$ whence $y \ni B$. On the other hand, if $K \forall A$ then $A \rightarrow B \in \mathrm{I}$ (Th $K) \subseteq x$. Both cases thus yield a contradiction, proving the desired.

Below we cite one of the main results of Goudsmit and Iemhoff, 2012, Theorem 1. The main point is that this perfectly characterizes when a model has an extension satisfying a given theory, and that this characterization works for logics extending minimal logic.

\section{Theorem}

Let $K$ be a Kripke model, let $x$ be a subset of Th $K$. The following are equivalent:

(i) the set $x \cup \mathrm{I}(\mathrm{Th} K)$ is saturated in Th $K$;

(ii) there is a tight predecessor of Th $K$ containing $x$ and I (Th $K)$;

(iii) there is a $y \subseteq \operatorname{Th} K$ such that $x \subseteq y=\operatorname{Th} K / y$.

\section{Extension Properties and Projectivity}

Every model of minimal logic has an extension, and the same goes for models of IPC (in both cases, forcing nothing at all will do). In the $n^{\text {th }}$ Gabby-de Jongh logic one can give an extension to any juxtaposition of $n+1$ many models, but more might fail. This property is of particular interest to us.

\section{Definition (Extension Properties)}

A theory $x$ is said to have the $n^{\text {th }}$ extension property when for each set $\mathcal{K}$ of $n$ rooted models of $x$ there exists an extension of $\amalg \mathcal{K}$ forcing $x$. Given another theory $y$, we say that $y$ has extensions over $x$ if for each model $K \Vdash y$ such that there is an extension forcing $x$, there is an extension forcing $y$.

The property of having extensions over a given base theory is quite interesting. First, there is a nice correspondence with projectivity in the sense of Ghilardi (1999). Projectivity and projective formulae in particular play a role in unification theory and in characterizing admissible rules, see for instance Ghilardi (1997) and Dzik (2011). Secondly, when $y$ has extensions over $x$ and $x$ has the $n^{\text {th }}$ extension property, so does $y$. 
The definition below is a modest generalization of projectivity in the sense of that paper. Note that a formula would be called projective there precisely if it were projective over the theorems of IPC.

\section{Definition (Projectivity)}

A set of formulae $y$ is said to be projective over $x$ when there is a substitution $\sigma$ (called the projective unifier) such that

$$
x \vdash \sigma(A) \text { for all } y \ni A \text { and } y \vdash \sigma(A) \leftrightarrow A \text { for all } A
$$

We can readily prove that projectivity over $x$ entails extensions over $x$. This specializes to the intermediate case in that projectivity over IPC entails the $n^{\text {th }}$ extension property for all $n$.

\section{Lemma}

Let $x \subseteq y$ be theories. Assume that $y$ is projective over $x$. Then $y$ has extensions over $x$.

Proof Take some Kripke model $K \Vdash y$ and assume that there exists an extension forcing $x$. By Theorem 2, this means that $x+\mathrm{I}(K) \preccurlyeq \mathrm{Th} K$. Per the same theorem, it suffices to prove that $y+\mathrm{I}(K) \preccurlyeq \operatorname{Th} K$. So suppose $y+\mathrm{I}(K) \vdash \bigvee \Delta$ for some finite non-empty $\Delta \subseteq \mathcal{L}$. This gives a finite $\Gamma \subseteq \mathrm{I}(K)$, which we can assume to be non-empty, such that $y \vdash \Gamma \Rightarrow \Delta$. Because $y$ is projective over $x$ we have a $\sigma$ such that $x \vdash \sigma(A)$ for all $A \in y$ and $y \vdash \sigma(A) \equiv A$ for all $A$, fix this $\sigma$. Transitivity ensures that $x \vdash \sigma(\Gamma) \Rightarrow \sigma(\Delta)$.

Observe that $\sigma(\Gamma) \subseteq \mathrm{I}(K)$, because if $A \rightarrow B \in \mathrm{I}(K)$ then $K \forall A$ and $K \Vdash A \rightarrow B$. Now as $y \subseteq$ Th $K$ it follows that $K \forall \sigma(A)$ and $K \Vdash \sigma(A \rightarrow B)$.

We now know $x \vdash \sigma(\Gamma) \Rightarrow \sigma(\Delta)$ and $x+\mathrm{I}(K) \vdash \sigma(C)$ for all $C \in \Gamma$, so the deduction theorem and transitivity ensures $x+\mathrm{I}(K) \vdash \bigvee \sigma(\Delta)$. The assumption $x+\mathrm{I}(K) \preccurlyeq$ Th $K$ now proves $K \Vdash \sigma(A)$ for some $A \in \Delta$, whence $K \Vdash A$ is readily derived. This proves the desired.

Do note that the above proof is basically the same as that of Ghilardi, 1999, Theorem 5, (ii) entails (iii), it is slightly rephrased in terms of our characterization of extensions, and isolates the bare necessities. The proof in the other direction unfortunately does not generalize as readily, this is still work in progress. We solely cite the easily generalizable part without proof.

\section{Lemma}

Assume that the logic at hand is an intermediate logic. Let $x$ be a theory with the finite model property and let $A$ be a formula such that $x+A$ has extensions over $x$. Now $x+A$ is projective over $x$.

We end this section with one observation on the use of projectivity towards providing bases of admissible rules. The nice property of projective formulae $A$ is that $A \sim B$ if and only if $A \vdash B .^{2}$ When $x+A$ admits all admissible rules of $x$, that is to say, when each rule is admissible with respect to $\vdash_{x+A}$ whenever it is admissible with respect to $\vdash_{x}$, we get the same nice property. Below we prove this, and moreover show that when $y$ is projective over $x$, it follows that $y$ admits all rules of $x$. This is interesting, as $y$ also "inherits $n^{\text {th }}$ extension properties" from $x$ as stated above.

\footnotetext{
${ }^{2}$ This must be very well-known as it is re-proven quite often, see for instance Cintula and Metcalfe (2010, Lemma 2.3), Iemhoff (2005, Section 2.6), Iemhoff and Metcalfe (2009a, Lemma 1.a), Iemhoff and Metcalfe (2009b, Lemma 6), Jeřábek (2005, Theorem 4.1) and Dzik (2011, Corollary 6).
} 


\section{Lemma}

Let $x$ be closed under substitution. Suppose that $A$ is such that all rules that are admissible for $x$ are admissible for $x+A$ too. Now $A \sim_{x} B$ if and only if $x+A \vdash B$.

Proof From left to right, assume that $A \sim_{x} B$. Now $A \sim_{x+A} B$ follows from assumption, whence the desired is immediate by monotonicity. Conversely, suppose that $x+A \vdash B$. Let $\sigma$ be arbitrary and additionally assume that $x \vdash \sigma(A)$. We now see that $x+\sigma(A) \supseteq \sigma(x)+\sigma(A) \vdash \sigma(B)$, so transitivity yields $x \vdash \sigma(B)$ as desired.

\section{Lemma}

Let $x \subseteq y$ be sets of formulae. If $y$ is projective over $x$ then $y$ admits all rules that $x$ admits.

Proof Let $\rho$ be the projective unifier of $y$ over $x$. Assume that $\Gamma \sim_{x} \Delta$, and suppose that $\sigma$ is such that $y \vdash \sigma(A)$ for all $\Gamma \ni A$. Because $\rho$ unifies $y$ under $x$ we get $x \vdash \rho(A)$ for all $y \ni A$, so by transitivity, $x \vdash \rho \sigma(A)$ for all $A \in \Gamma$. Now note that as $\Gamma \sim_{x} \Delta$, we obtain a $A \in \Delta$ such that $x \vdash \rho \sigma(A)$. As $x \subseteq y$ we obtain $y \vdash \sigma(A)$ as desired.

\section{Admissible Rules}

The interesting scheme of admissible rules of choice are the Visser rules. They have been shown to be a basis of admissibility for IPC, and they correspond nicely to the extension property. To neatly restrict the above result to the $n^{\text {th }}$ extension property the de Jongh rules were introduced in Goudsmit and Iemhoff (2012). With the machinery available here we can quite smoothly prove that the de Jongh rules are admissible in any of the logics at hand that satisfy the disjunction property. Let us first define these rules. As an auxiliary definition, say that a non-empty set $\mathcal{U}$ is an $n$-cover of another set $X$ if $\cup \mathcal{U}=X, \emptyset \notin \mathcal{U}$ and $|\mathcal{U}| \leq n$. Per natural number $n$, finite non-empty set of implications $\Gamma$, non-empty finite set $\Delta$ and $n$-cover $\mathcal{U}$ of $\Gamma^{\mathrm{a}}$, the $n^{\text {th }}$ de Jongh rule determined by $\Gamma, \Delta$ and $\mathcal{U}$ is defined as below.

$$
\frac{\Gamma \Rightarrow \Delta}{\{\Gamma \Rightarrow \Theta \mid \Theta \in \mathcal{U} \cup \Delta\}} \mathcal{U} \text { is a } n \text {-cover of } \Gamma^{\mathrm{a}}
$$

\section{Lemma}

Assume that $x$ has the disjunction property and the $n^{\text {th }}$ extension property. Now $x$ admits the $n^{\text {th }}$ de Jongh rule.

Proof Suppose we have non-empty finite $\Gamma$ (with only implications) and $\Delta$ (arbitrary) and an $n$-cover $\mathcal{U}$ of $\Gamma^{\mathrm{a}}$ such that $x \vdash \Gamma \Rightarrow \Delta$ but $x \nvdash \Gamma \Rightarrow \Theta$ for any $\Theta \in \mathcal{U} \cup \Delta$. Completeness provides us with rooted models $K_{U}$ for each $U \in \mathcal{U}$ such that $K_{U} \Vdash x+\Gamma$ but $K_{U} \Vdash \forall U$. Furthermore, we have models $K_{A}$ for each $A \in \Delta$ such that $K_{A} \Vdash x+\Gamma$ but $K_{A} \Vdash \forall$.

By the $n^{\text {th }}$ extension property we have a model $K_{\mathcal{U}}$ extending $\coprod_{u \in \mathcal{U}} K_{U}$ such that $K_{\mathcal{U}} \Vdash x$. The disjunction property of $x$ ensures us a rooted model $K \Vdash x$ of which $K_{\mathcal{U}}$ and all of $K_{A}$ for $A \in \Delta$ are open subsets. 
Suppose that $A \rightarrow B \in \Gamma$. If $K \Vdash A$ then pick some $A \in U \in \mathcal{U}$ and note that $K_{U} \Vdash A \vdash \bigvee U$, a contradiction. This entails that $K \Vdash x+\Gamma$. As $x+\Gamma \vdash \Delta$ we know that $K \Vdash \bigvee \Delta$. This gives a $A \in \Delta$ such that $K \Vdash A$, which entails $K_{A} \Vdash A$, quod non. Hence all $n^{\text {th }}$ de Jongh rules are admissible.

The de Jongh rules have quite a lot of parameters, it would be nice if these could be restricted in number. A nice source of inspiration can be found in the rule below, which has been studied in several incarnations before. Its admissibility for singleton covers with $n=2$ in IPC was discussed by Mints (1976). Skura (1989) considered this rule, also with only singleton covers but for arbitrary $n$, and proved that IPC is the sole intermediate logic which admits them all. Per natural number $n$, non-empty finite set of implications $\Gamma$ and $n$-cover $\mathcal{U}$ of $\Gamma^{\mathrm{a}}$ we define the $n^{\text {th }}$ Mints-Skura rule as below.

$$
\frac{\Gamma \Rightarrow \Gamma^{\mathrm{a}}}{\{\Gamma \Rightarrow \Theta \mid \Theta \in \mathcal{U}\}} \mathcal{U} \text { is a } n \text {-cover of } \Gamma^{\mathrm{a}}
$$

The rule clearly is a special case of the de Jongh rule, so it holds in the presence of the $n^{\text {th }}$ extension property due to Lemma 9 . We prove the the converse below.

\section{Lemma}

Assume that $x$ has the disjunction property and assume that $x$ admits the $n^{\text {th }}$ Mints-Skura rule. Now $x$ has the $n^{\text {th }}$ extension property.

Proof Consider a set $\mathcal{K}$ of $n$ rooted models of $x$. Due to Theorem 2, the model $L:=\coprod \mathcal{K}$ has an extension precisely if $x+\mathrm{I}$ ( Th $L) \preccurlyeq \mathrm{Th} L$. We reason by contradiction, so suppose that $x+\mathrm{I}(\mathrm{Th} L) \vdash$ $\bigvee \Delta$ yet $\operatorname{Th} L \cap \Delta$ is empty for some finite non-empty $\Delta$. The latter ensures that $\Delta \subseteq \mathrm{I}(\mathrm{Th} L)^{\mathrm{a}}$, the former ensures some finite non-empty $\Gamma \subseteq \mathrm{I}(\operatorname{Th} L)$ such that $x+\Gamma \vdash \bigvee \Delta$. Monotonicity ensures that we can assume that $\Delta \subseteq \Gamma^{\mathrm{a}}$ without loss of any generality. We thus know that $x+\Gamma \vdash \bigvee \Gamma^{\mathrm{a}}$. Construct the cover $\mathcal{U}$ as below, clearly of size at most $n$. It does not contain the empty set by construction, and its union equals $\Gamma^{\mathrm{a}}$, so $\mathcal{U}$ is not empty as well.

$$
\mathcal{U}:=\left\{\Theta_{K}=\Gamma^{\mathrm{a}}-\operatorname{Th} K \mid K \in \mathcal{K}\right\}-\{\emptyset\}
$$

Due to the $n^{\text {th }}$ Mints-Skura rule at $\mathcal{U}$ we now know that $x \vdash \Gamma \Rightarrow \Theta_{K}$ for some $K \in \mathcal{K}$. Now see that as $L \Vdash x$ and $L \Vdash \mathrm{I}(\operatorname{Th} L) \supseteq \Gamma$ we know that $L \Vdash \bigvee \Theta_{K}$. This ensures that $K \Vdash \bigvee \Theta_{K}$, whence Th $K$ must have a non-empty intersection with $\Theta_{K}=\Gamma^{\mathrm{a}}-$ Th $K$, utter nonsense of course.

The above two lemmas immediately entail the theorem below. Iemhoff (2001a, Lemma 3.3) argued that any intermediate logic with the $n^{\text {th }}$ extension property for all $n$ must be IPC. So the theorem below re-proves the main result of Skura (1989), namely that IPC is the sole intermediate logic which admits all Mints-Skura rules.

\section{Theorem}

Each logic above minimal logic with the disjunction property admits the $n^{\text {th }}$ Mints-Skura rule if and only if it has the $n^{\text {th }}$ extension property. 


\section{References}

Aczel, Peter H.G. (1968). "Saturated Intuitionistic Theories". In: Contributions to Mathematical Logic Proceedings of the Logic Colloquium, Hannover 1966. Ed. by K. Schütte H. Arnold Schmidt and H.-J. Thiele. Vol. 50. Studies in Logic and the Foundations of Mathematics. Elsevier, pp. 111. DOI: $10.1016 / \mathrm{S} 0049-237 \mathrm{X}(08) 70515-9$.

Chagrov, Alexander and Michael Zakharyashchev (1991). "The Disjunction Property of Intermediate Propositional Logics". In: Studia Logica: 50.2, pp. 189-216. ISSN: 00393215. DOI: 10.1007/ BF00370182.

Cintula, Petr and George Metcalfe (2010). "Admissible rules in the implication-negation fragment of intuitionistic logic". In: Annals of Pure and Applied Logic 162.2, pp. 162-171. ISSN: 0168-0072. DOI: $10.1016 /$ j.apal.2010.09.001.

Citkin, Alexander (2012). "A note on admissible rules and the disjunction property in intermediate logics". In: Archive for Mathematical Logic 51 (1), pp. 1-14. ISSN: 0933-5846. DOI: 10.1007/ s00153-011-0250-y.

Došen, Kosta (1989). "Sequent-systems and groupoid models. II". In: Studia Logica 48.1 (1), pp. 4165. ISSN: 0039-3215. DOI: 10.1007/BF00370633.

Dzik, Wojciech (2011). "Remarks on Projective Unifiers". In: Bulletin of the Section of Logic 40.1, pp. $37-45$.

Friedman, Harvey (1975). "One Hundred and Two Problems in Mathematical Logic". In: The Journal of Symbolic Logic 40.2, pp. 113-129. ISSN: 00224812. JSTOR: 2271891.

Gabbay, Dov M. and Dick H.J. de Jongh (1974). "A Sequence of Decidable Finitely Axiomatizable Intermediate Logics with the Disjunction Property". In: The Journal of Symbolic Logic 39.1, pp. 67-78. ISSN: 00224812. JSTOR: 2272344.

Ghilardi, Silvio (1997). "Unification through Projectivity". In: Journal of Logic and Computation 7.6, pp. $733-752$. DOI: $10.1093 / \log c o m / 7.6 .733$.

- (1999). "Unification in Intuitionistic Logic". In: The Journal of Symbolic Logic 64.2, pp. 859880. ISSN: 00224812. JSTOR: 2586506.

Goudsmit, Jeroen P. and Rosalie Iemhoff (2012). "On unification and admissible rules in Gabbay-de Jongh logics". In: Logic Group Preprint Series 297, pp. 1-18. ISSN: 0929-0710.

Iemhoff, Rosalie (2001a). "A(nother) characterization of intuitionistic propositional logic". In: $A n$ nals of Pure and Applied Logic 113.1-3. First St. Petersburg Conference on Days of Logic and Computability, pp. 161-173. ISSN: 0168-0072. DOI: 10.1016/S0168-0072(01)00056-2.

- (2001b). "On the Admissible Rules of Intuitionistic Propositional Logic". In: The Journal of Symbolic Logic 66.1, pp. 281-294. ISSN: 00224812. JSTOR: 2694922.

- (2005). "Intermediate Logics and Visser's Rules". In: Notre Dame Journal of Formal Logic 46.1, pp. 65-81. DOI: $10.1305 / \mathrm{ndjfl} / 1107220674$.

Iemhoff, Rosalie and George Metcalfe (2009a). "Hypersequent Systems for the Admissible Rules of Modal and Intermediate Logics". In: Logical Foundations of Computer Science. Ed. by Sergei Artemov and Anil Nerode. Vol. 5407. Lecture Notes in Computer Science. Springer Berlin / Heidelberg, pp. 230-245. ISBN: 978-3-540-92686-3. DOI: 10.1007/978-3-540-92687-0_16.

- (2009b). "Proof theory for admissible rules". In: Annals of Pure and Applied Logic 159.1-2, pp. 171-186. ISSN: 0168-0072. DOI: 10.1016/j.apal.2008.10.011.

Jeřábek, Emil (2005). "Admissible Rules of Modal Logics". In: Journal of Logic and Computation 15.4, pp. 411-431. DOI: 10.1093/logcom/exi029. 
Johansson, Ingebrigt (1937). "Der Minimalkalkül, ein reduzierter intuitionistischer Formalismus". In: Compositio Mathematica 4, pp. 119-136.

Johnstone, Peter T. (1982). Stone Spaces. Ed. by D.J.H Garling et al. Vol. 3. Cambridge studies in advanced mathematics. Cambridge University Press. ISBN: 0-521-23893-5.

Lorenzen, Paul (1955). Einführung in die operative logik und mathematik. Vol. 78. Die Grundlehren der mathematischen Wissenschaften in Einzeldarstellungen. Springer-Verlag.

Maksimova, Larisa L. (1986). "On Maximal Intermediate Logics with the Disjunction Property". English. In: Studia Logica: An International Journal for Symbolic Logic 45.1, pp. 69-75. ISSN: 00393215 . JSTOR: 20015248.

Mints, Grigori E. (1976). "Derivability of admissible rules". In: Journal of Mathematical Sciences 6 (4), pp. 417-421. ISSN: 1072-3374. DOI: 10.1007/BF01084082.

Odintsov, Sergei and Vladimir V. Rybakov (2012). "Unification and Admissible rules for paraconsistent minimal Johanssons' logic J and positive intuitionistic logic IPC+". Submitted to Annals of Pure and Applied Logic in Feburary 2012.

Rozière, Paul (1992). "Règles admissibles en calcul propositionnel intuitionniste". PhD thesis. Université de Paris VII.

Rybakov, Vladimir V. (1984). "A criterion for admissibility of rules in the model system S4 and the intuitionistic logic". In: Algebra and Logic 23 (5), pp. 369-384. ISSN: 0002-5232. DOI: 10.1007/ BF01982031.

- (1997). Admissibility of Logical Inference Rules. Vol. 136. Studies in Logic and the Foundations of Mathematics. Elsevier. ISBN: 978-0-444-89505-9.

Skura, Thomasz (1989). "A complete syntactical characterization of the intuitionistic logic". In: Reports on Mathematical Logic 23, pp. 75-80.

Smoryński, Craig (1973). "Applications of Kripke models". In: Metamathematical Investigation of Intuitionistic Arithmetic and Analysis. Ed. by Anne Sjerp Troelstra. Vol. 344. Lecture Notes in Mathematics. Springer Berlin / Heidelberg, pp. 324-391. DOI: 10.1007/BFb0066744.

Troelstra, Anne Sjerp and Dirk van Dalen (1988). "Logic". In: Constructivism in Mathematics - An Introduction. Vol. 121. Studies in Logic and the Foundations of Mathematics. Elsevier, pp. 35111. DOI: $10.1016 / \mathrm{S} 0049-237 \mathrm{X}(09) 70527-0$. 